\title{
A survey of dentists' practice in the restoration of the shortened dental arch
}

\author{
Mohammad-Zakaria Nassani ${ }^{1}$, Hugh Devlin ${ }^{2}$, Bassel Tarakji ${ }^{3}$, James-Fraser McCord ${ }^{4}$ \\ ${ }^{1}$ Lecturer in Prosthetic Dentistry, Department of Removable Prosthodontics, Faculty of Dentistry, University of Aleppo, Syria \\ ${ }^{2}$ Professor of Restorative Dentistry, School of Dentistry, University of Manchester, UK \\ ${ }^{3}$ Lecturer in oral medicine, Faculty of Dentistry, University of Aleppo, Syria \\ ${ }^{4}$ Professor of Restorative Dentistry and Honorary Consultant in Restorative Dentistry, Glasgow Dental Hospital and School, UK
}

Correspondence:

P O Box 3417,

Aleppo - Syria

mznassani@hotmail.com

Received: 12/04/2009

Accepted: 08/06/2009

\author{
Nassani MZ, Devlin H, Tarakji B, McCord JF. A survey of dentists' prac- \\ tice in the restoration of the shortened dental arch. Med Oral Patol Oral \\ Cir Bucal. 2010 Jan 1;15 (1):e85-9. \\ http://www.medicinaoral.com/medoralfree 01/v15i1/medoralv15ilp85.pdf

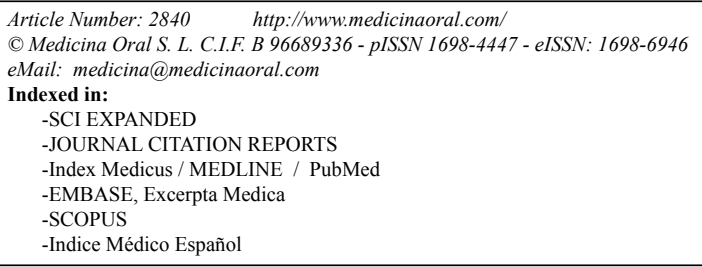

\begin{abstract}
Objectives: The aim of this survey was to determine how a sample of dentists working in general dental practice in the UK sought to restore the mouths of patients who had shortened dental arches (SDAs). Study design: A survey was conducted over a period of six months on four commercial dental laboratories. Cases of SDAs comprising the anterior teeth and 2 to 4 premolars were examined; dental prescriptions were scrutinized and a special data collection form was completed accordingly. Results: A total of 140 SDA cases were examined. Most of these cases were for mandibular SDAs (88.6\%). Of the recorded SDA cases, $67.2 \%$ were restored by cobalt-chromium based removable partial dentures (RPD); $25.7 \%$ were extended by acrylic-resin based RPDs; implants were provided to restore 8 SDA cases (5.7\%); and only two SDA cases $(1.4 \%)$ were extended by cantilevered fixed bridges. Neither the gender of the patient $([\mathrm{Chi}(2)=4.19, \mathrm{p}>0.05)$ nor the length of the SDA ([Chi $(2)=6.51, \mathrm{p}>0.05)$ influenced the choice of prosthesis to be implemented. Conclusions: It would appear from the results of this survey that among the different restorative treatment options for SDA, the RPD was the most popular treatment option for dentists surveyed in this UK study. Extending the SDA by either an implant-supported prosthesis or cantilevered fixed bridges did not appear to be a popular choice of treatment.
\end{abstract}

Key words: Survey, shortened dental arch, partial dentures, implants, cantilevered bridges.

\section{Introduction}

For patients with SDAs, many treatment options are available. The existing situation can be maintained by stabilizing the present dentition and improving the occlusion without extending the arch. In other words, all treatment efforts should be concentrated on preserving sound anterior and premolar teeth and avoiding extensive restorative treatment in the molar regions. Alternatively, the SDA can be extended by either a free-end saddle removable partial denture (FESRPD), cantilevered fixed bridge or by an implant-supported prosthesis (1).

Clinical studies indicated that SDAs comprising the anterior and premolar teeth, appeared to have, in the long term, sufficient adaptive capacity to ensure adequate oral function in terms of chewing ability, aesthetics, stability of the dentition, temporomandibular joint (TMJ) function and functional habits (2). However, in 
many cases the SDA therapy is contraindicated. Also, some patients may be reluctant to leave their SDAs unrestored. Furthermore, there are still situations where extending the SDA should be considered, where loss of posterior teeth creates, for example, aesthetic problems, occlusal instability or chewing difficulties $(3,4)$. The decision about what would provide the most acceptable treatment option, functionally and aesthetically, depends on a variety of factors. Each treatment option for SDA patients has, in some respects, unsatisfactory elements. Furthermore, these options should be approached carefully after thorough assessment and detailed treatment plan. The resultant problems in treatment decision-making in this situation have resulted in it being called "a prosthodontic dilemma" (5). To date, on the level of clinical practice, it is not yet clear what is the optimal treatment in the management of the SDAs. The aim of this survey was to determine how a sample of dentists working in general dental practice in the UK sought to restore mouths of patients who had reduced or SDAs.

\section{Material and Methods}

Over a period of six months, a survey of four commercial dental laboratories was conducted. These laboratories were located in three towns in Northern England and all of them received work from all around the UK. Two of the laboratories undertake only private work and two of them undertake both National Health Service and private work. All four dental laboratories were visited frequently by the first author (at least twice a month). In each visit, dental casts with SDAs comprising the anterior teeth and 2 to 4 premolars (standing or restored) were examined. Dentists' prescriptions were scrutinized and a special data collection form was completed accordingly.

Casts with significant anterior interdental spaces (i.e wider than the width of half a premolar) were excluded. Cases with a SDA in both jaws were recorded in two separate forms i.e. each jaw was considered a separate prescription. When the treatment requested for the SDA was an immediate denture, the case was excluded, as this was perceived to be a transitional treatment option leading to a definitive edentulous treatment option. Cases prescribed for cobalt-chromium based RPDs were examined only after the trial insertion stage and subsequent to fabrication of the metal framework. Similarly, where the acrylic-resin based RPDs were prescribed, these were checked only following the trial insertion phase. After the end of the trial insertion stage no further design instructions could be provided by the dentist and one could be certain that no relevant information was missed.

\section{Results}

By the end of the survey a total of 140 cases were examined, and these were sent from across the UK. However, the number of cases from England was far more than any other of the continental countries of the UK ( $80.7 \%)$ (Table 1).

Table 1. Distribution of SDA cases according to practice location.

\begin{tabular}{|l|c|c|}
\hline Practice location & Frequency & Percent \\
\hline England & 113 & 80.7 \\
\hline Scotland & 10 & 7.2 \\
\hline Wales & 8 & 5.7 \\
\hline Northern Ireland & 1 & 0.7 \\
\hline Isle of Man & 1 & 0.7 \\
\hline Missing & 7 & 5.0 \\
\hline Total & 140 & 100.0 \\
\hline
\end{tabular}

The proportion of cases for female patients was higher than for males (51.4\% compared to $29.3 \%$ ), but there was no evidence about the patient's gender in about one fifth of the prescriptions (19.3\%). The majority of the surveyed cases were mandibular SDAs (88.6\%). Besides, there was a greater proportion of the cases which had a SDA length from the first premolar to the first premolar $(42.1 \%)$ than from the first premolar to the second premolar (35\%). SDAs with standing teeth from the second premolar to the second premolar were the least frequent $(22.9 \%)$. The nature of the opposing dentition varied from case to case. In $25 \%$ of the cases the opposing dental arch was restored by a cobalt-chromium based RPD, $22.1 \%$ by an acrylic-resin based RPD and, in $15 \%$, by a complete denture. The opposing dentition had unrestored gaps in $17.2 \%$ of the examined cases. These spaces were left without any kind of prosthodontic restoration at the time of this survey. The opposing dentition was a complete dental arch in $13.6 \%$ of the cases. In only two cases $(1.4 \%)$ the opposing dentition was restored by an overdenture. The opposing model was not available in the laboratory in 8 cases $(5.7 \%)$. The survey showed that the majority of the recorded SDAs (67.2\%) were restored by a cobalt-chromium based RPD. Approximately one quarter of the cases were restored by an acrylic-resin based RPD and a few cases $(\mathrm{N}=8$, 5.7\%) were restored by an implant-supported prosthesis. Extending the SDA by a cantilevered fixed bridge was uncommon practice (Table 2). Neither the gender of the patient $(\mathrm{X} 2=4.19, \mathrm{df}=3, \mathrm{p}=0.242)$ nor the length of the SDA $(\mathrm{X} 2=6.51, \mathrm{df}=6, \mathrm{p}=0.368)$ appeared to have influenced the treatment choice in the restoration of the SDAs. 
Table 2. Type of prosthodontic restoration requested for the SDA.

\begin{tabular}{|l|c|c|}
\hline & Frequency & Percent \\
\hline Cobalt-chromium based RPD & 94 & 67.2 \\
\hline Acrylic-resin based RPD & 36 & 25.7 \\
\hline Implant prosthesis & 8 & 5.7 \\
\hline Cantilevered fixed bridge & 2 & 1.4 \\
\hline Total & 140 & 100.0 \\
\hline
\end{tabular}

Almost all the requested cobalt-chromium based RPDs were retained by conventional direct retainers (clasps) - 96.8\%. Precision attachments were used as a retentive element in two cases (2.1\%) and the attachments used were of the extracoronal type. Only one telescopic crown-retained partial denture was requested during the survey. The prescriptions of dentists who requested clasp-retained RPDs were checked in order to assess the level of dependence on the dental technician in the design of the RPDs. It was found that dentists' prescriptions did not have any instructions about the design of the requested partial denture in a considerable proportion of the cases $(31.9 \%)$.

The majority of the 36 acrylic-resin based RPDs in this survey, were mandibular dentures $(\mathrm{N}=30,83.3 \%)$. Analysis of dentists' prescriptions for the acrylic-resin based RPD indicated that dentists instructed the technician to construct a denture with clasps in 12 out of the 36 recorded cases (33.3\%). Occlusal rests (wrought type) were requested in one case $(2.8 \%)$ and the lingual bar was requested in two out of the 30 mandibular treated cases $(6.7 \%)$. In both cases the request was for wrought stainless steel lingual bar.

Two out of the eight implant cases were in the maxillary arch. In only one of these cases, the dentist planned the support to be shared between teeth and implants. The seven other cases were planned to gain their support from the implants alone. In no case did the dentists request that an implant-supported denture be constructed, in all cases, implant-supported crown or bridgework was prescribed.

The two cantilevered bridges recorded in this survey were in the mandible and were of a conventional type. No posterior cantilevered fixed resin-bonded bridges (RBBs) were prescribed by any of the clinicians over the period of this survey.

\section{Discussion}

Firstly, it must be noted that the dental laboratories visited over this survey were chosen on basis of practicality and were in no way intended to be representative of the British dental laboratories in general. Yet the examined SDA cases were sent from across the UK, the results of this survey cannot be generalized to reflect the current trends in the restoration of the SDAs in general dental practice in the UK.

In this survey, the vast majority of the collected cases were for mandibular SDAs (88.6\%); while it could be said that this is a finding peculiar to this study, it is in accordance with the results of previous investigations. The study of Spratley (6) indicated that loss of posterior teeth is far more common in the mandible than in maxilla. Other studies conducted in different countries indicated a significantly higher incidence of free-end saddles with unbroken anterior dentition in the mandible than in the maxilla (7-9). The results of this study showed that SDAs with 8 remaining anterior teeth were the most frequent (42.1\%). On the contrary, SDAs with 10 remaining anterior teeth were the least frequent $(22.9 \%)$. It appears that in our sample the demand for active prosthodontic treatment of the SDA increased with the decrease in the length of the SDA.

It is well documented in the literature that treatment by a FESRPD is problematic. This is because the planning of a FESRPD may present the dentist with difficulty in achieving the aim of providing a satisfactory and comfortable denture because of the different and differing responses of teeth and mucosa to occlusal loads and the associated clinical problems relating to support and stability (10). Clinical surveys indicated a high failure rate of FESRPDs especially in the mandible $(11,12)$. Kayser et al. (3) considered the prosthodontic intervention to extend the SDA by a FESRPD to be a kind of "overtreatment". The same authors believed that such treatment does not contribute to the maintenance of a healthy, natural functioning dentition for life. By contrast, the provision of a FESRPD may introduce unfavourable conditions for the remaining dentitions. Clinical trials by the Nijmegen group questioned the contribution of FESRPDs to oral functions (2). Their work indicated that extending an SDA consisting of 3 to 5 occlusal units by a FESRPD did not lead to an evident improvement in oral function in terms of chewing ability, oral comfort, aesthetics, stability of the dentition, TMJ function and functional habits (2). Witter and associates (13) challenged what was a strongly held view for the rationale of providing RPDs. They stated that undertaking conventional removable prosthodontic intervention as a routine preventive measure in SDAs to avoid occlusal collapse, should be discouraged. Jepson et al.(14) found that patients' compliance with wearing partial dentures was significantly related to the presence of anterior replacement teeth. Dentures that were never worn were more likely to have no replacement anterior teeth. Furthermore, patients place little value on RPDs that replaced missing molar teeth in an SDA (15). In a recent review of the literature the old dogma of prescribing the RPD on prophylactic ground, i.e to prevent 
disorder of oral functions, was questioned. The authors indicated that the feasibility to plan the restorative dental care according to the SDA concept should preclude the indication for an RPD (16).

Although the prognosis of any FESRPD is not predictable and its contribution to oral functions in patients with SDAs is considered to be dubious, the present survey indicated that the RPD is the most popular treatment option for patients with SDAs among general dental practitioners in our study sample. This may be attributable to economic considerations (16) as such treatment is relatively cheap, simple, non-invasive and within the skills and experience of most dentists. However, in the long term, the costs of maintenance and repair and the potential for failure should be considered, indeed it could be argued that a study into the cost-effectiveness of RPD treatment for patients with SDAs is required.

The results of this study show that, in our study sample, the popularity of the cobalt-chromium based RPD far exceeded that of the acrylic-resin based RPD. This finding is at odds with published trends by the Dental Practice Board digest (17), which reported that the number of acrylic-resin based RPDs provided in general dental practice in England and Wales far surpassed the number of cobalt-chromium based RPDs. This finding could be explained by the fact that most of the restored SDAs in this survey were in the mandible where the use of the tissue-borne acrylic-resin based RPD is expected to be more damaging to oral tissues, less stable and less comfortable if compared with the use of the tooth and mucosa-borne cobalt-chromium based RPD (12).

The use of precision attachments, milled or telescopic crowns as retentive elements for the metal-based FESRPD was uncommon in the cases observed in this study. Despite the supposedly aesthetic superiority of precision attachments, milled or telescopic crowns to metal clasps, the clasp-retained RPD seems to be the most popular type of cobalt-chromium based RPD in general dental practice in the UK. The higher biological and monetary cost coupled with the more complex clinical and technical procedures involved in making a partial denture with precision attachments, milled or telescopic crowns appear to discourage their clinical practice.

Scrutinising dentists' prescriptions indicated that delegating the design of the cobalt-chromium based RPD entirely to the dental technician is a common practice among a considerable proportion of the dentists in this survey $(31.9 \%)$. This is in line with previous surveys $(18,19)$ and warranty further investigations to underline reasons behind such kind of malpractice.

It is well known that implant treatment is relatively complicated in terms of planning, preparation and delivery. Furthermore, it is a costly treatment option. In this survey, the request for implant treatment was very limited (5.7\%). Implant treatment appears to be uncom- mon in the restoration of the SDAs. This could further support the arguments that barriers to implant treatment may overwhelm its advantages and restrict its practice. It has to be stated, however, that in the future dental implants may be seen as a highly cost-effective alternative. Although initial costs are high, early success rates are also high.

Randomised controlled trials showed that cantilevered fixed RBBs offered an alternative treatment option to conventional mandibular RPDs at a lower biological price. Moreover, this treatment modality seems to be an effective treatment option for patients with SDAs in terms of comfort and acceptance (20-22). However, this investigation indicated that extending the SDA by cantilevered fixed bridges is a very rare dental practice among dentists in this survey. This may reflect a negative attitude to the value of cantilevered bridges in the restoration of the SDA. Extending the SDA by cantilevered RBBs was not a recorded choice. Further work is therefore required to explore the real motive behind this clinical behaviour, perhaps by a detailed interview questionnaire of dentists.

Within the limitations of this study, it seems that the RPD is the most popular restoration for the SDAs among the sample of dentists surveyed in this investigation. Extending the SDA by either implant-supported prosthesis or cantilevered fixed bridges appear to be uncommon practice. Further work with a representative sample of the British general dental practitioners is required to confirm the findings of this survey.

In an era where patients' values and preferences are central to optimal treatment decision making, future research should be directed towards studying the impact of the different treatment options for the SDA on oral health related quality of life, patients' satisfaction and patients' utilities. A cost-effectiveness analysis is very important in this respect. The outcome of such research may impact upon future trends in the treatment of the SDA.

\section{References}

1. Kayser AF. Shortened dental arch: a therapeutic concept in reduced dentitions and certain high-risk groups. Int J Periodontics Restorative Dent. 1989;9:426-49.

2. Kanno T, Carlsson GE. A review of the shortened dental arch concept focusing on the work by the Käyser/Nijmegen group. J Oral Rehabil. 2006;33:850-62.

3. Käyser AF, Witter DJ, Spanauf AJ. Overtreatment with removable partial dentures in shortened dental arches. Aust Dent J. 1987;32:17882 .

4. Allen PF, Witter DF, Wilson NH, Kayser AF. Shortened dental arch therapy: views of consultants in restorative dentistry in the United Kingdom. J Oral Rehabil. 1996;23:481-5.

5. Devlin H. Replacement of missing molar teeth--a prosthodontic dilemma. Br Dent J. 1994;176:31-3.

6. Spratley MH. Posterior edentulousness and the prescription of partial dentures. Aust Dent J. 1988;33:43-6.

7. Owall BE, Taylor RL. A survey of dentitions and removable partial 
dentures constructed for patients in North America. J Prosthet Dent. 1989;61:465-70.

8. Owall G, Bieniek KW, Spiekermann H. Removable partial denture production in western Germany. Quintessence Int. 1995;26:621-7.

9. Owall B, Junggreen L, Yemm R. Removable partial denture production in Scotland. Quintessence Int. 1996;27:809-15.

10. Nairn RI. The problem of free-end denture bases. J Prosthet Dent. 1966;16:522-32.

11. Roberts BW. A survey of chrome-cobalt partial dentures. N Z Dent J. 1978;74:203-9.

12. Wetherell JD, Smales RJ. Partial denture failures: a long-term clinical survey. J Dent. 1980;8:333-40.

13. Witter DJ, Creugers NH, Kreulen CM, De Haan AF. Occlusal stability in shortened dental arches. J Dent Res. 2001;80:432-6.

14. Jepson NJ, Thomason JM, Steele JG. The influence of denture design on patient acceptance of partial dentures. Br Dent J. 1995;178:296-300.

15. Nassani MZ, Devlin H, McCord JF, Kay EJ. The shortened dental arch--an assessment of patients' dental health state utility values. Int Dent J. 2005;55:307-12.

16. Wöstmann B, Budtz-Jørgensen E, Jepson N, Mushimoto E,
Palmqvist S, Sofou A, et al. Indications for removable partial dentures: a literature review. Int J Prosthodont. 2005;18:139-45.

17. Dental Practice Board. 2001 - 2002 digest of statistics. Eastbourne: Dental Practice Board, 2002.

18. Basker RM, Harrison A, Davenport JC, Marshall JL. Partial denture design in general dental practice--10 years on. Br Dent $\mathrm{J}$. 1988;165:245-9.

19. Lynch CD, Allen PF. A survey of chrome-cobalt RPD design in Ireland. Int J Prosthodont. 2003;16:362-4.

20. Jepson NJ, Moynihan PJ, Kelly PJ, Watson GW, Thomason JM. Caries incidence following restoration of shortened lower dental arches in a randomized controlled trial. Br Dent J. 2001;191:140-4. 21. Jepson N, Allen F, Moynihan P, Kelly P, Thomason M. Patient satisfaction following restoration of shortened mandibular dental arches in a randomized controlled trial. Int J Prosthodont. 2003;16:409-14.

22. Thomason JM, Moynihan PJ, Steen N, Jepson NJ. Time to survival for the restoration of the shortened lower dental arch. J Dent Res. 2007;86:646-50. 